\title{
Computational modeling of shear forces and experimental validation of endothelial cell responses in an orbital well shaker system
}

\author{
Nenad Filipovic $^{\mathrm{a} *}$, Kedar Ghimire $^{\mathrm{b}}$, Igor Saveljic $^{\mathrm{a}}$, Zarko Milosevic $^{\mathrm{a}}$ and Curzio Ruegg ${ }^{\mathrm{b}}$ \\ ${ }^{a}$ Department for Applied Mechanics and Automatic Control, Faculty of Engineering, Center for Bioengineering, University of \\ Kragujevac, Sestre Janjic 6, Kragujevac 34000, Serbia; ${ }^{b}$ Department of Medicine, Faculty of Science, University of Fribourg, Fribourg,
} Switzerland

\begin{abstract}
Vascular endothelial cells are continuously exposed to hemodynamic shear stress. Intensity and type of shear stress are highly relevant to vascular physiology and pathology. Here, we modeled shear stress distribution in a tissue culture well $(R=17.5 \mathrm{~mm}$, fill volume $2 \mathrm{ml})$ under orbital translation using computational fluid dynamics with the finite element method. Free surface distribution, wall shear stress, inclination angle, drag force, and oscillatory index on the bottom surface were modeled. Obtained results predict nonuniform shear stress distribution during cycle, with higher oscillatory shear index, higher drag force values, higher circular component, and larger inclination angle of the shear stress at the periphery of the well compared with the center of the well. The oscillatory index, inclination angle, and drag force are new quantitative parameters modeled in this system, which provide a better understanding of the hydrodynamic conditions experienced and reflect the pulsatile character of blood flow in vivo. Validation experiments revealed that endothelial cells at the well periphery aligned under flow and increased Kruppel-like Factor 4 (KLF-4), cyclooxygenase-2 (COX-2) expression and endothelial nitric oxide synthase (eNOS) phosphorylation. In contrast, endothelial cells at the center of the well did not show clear directional alignment, did not induce the expression of KLF-4 and COX-2 nor increased eNOS phosphorylation. In conclusion, this improved computational modeling predicts that the orbital shaker model generates different hydrodynamic conditions at the periphery versus the center of the well eliciting divergent endothelial cell responses. The possibility of generating different hydrodynamic conditions in the same well makes this model highly attractive to study responses of distinct regions of the same endothelial monolayer to different types of shear stresses thereby better reflecting in vivo conditions.
\end{abstract}

Keywords: shear stress; modeling; endothelial cells; flow; orbital shaker

\section{Introduction}

Vascular endothelial cells are exposed to the hemodynamic forces of the blood at the luminal surface of the vessel, including circumferential stretch, hydrostatic pressure, and shear stress (Ballermann et al. 1998; Sumpio et al. 2002). Steady laminar shear stress has been shown to inhibit endothelial cell activation, proliferation, apoptosis, and to promote the expression of atheroprotective genes (Chiu and Chien 2011). In contrast, low, oscillatory, or turbulent shear stress is considered to favor endothelial cell activation, inflammation and to promote atherogenesis (Paszkowiak and Dardik 2003). Shear stress is also an important parameter that controls vascular remodeling and arteriogenesis (Cai and Schaper 2008).

Cultured endothelial cells are routinely studied under static conditions (Folkman et al. 1979; Schor et al. 1983). It is well known that endothelial cells grown under static conditions may not be representative of endothelial cells in the body where they are constantly subjected to fluid forces from the blood flow (Nerem et al. 1998; Warboys et al. 2010). Increasing evidence indicates that shear stress modulates cell autonomous, paracrine and endocrine functions of endothelial cells (Nerem et al. 1998). For example, expression of cyclooxygenase-2 (COX-2), a key enzyme in the synthesis of prostaglandins involved in the regulation of vascular homeostasis and inflammation, and activation of endothelial nitric oxide synthase (eNOS), an enzyme controlling vasomotion through the production of nitric oxide (NO), are regulated by shear stress (Topper et al. 1996).

Two main types of devices are commonly used for the generation of shear stress in tissue culture conditions. The first one consists of parallel plates forming a 'flow chamber' through which medium is pumped to generate shear stress on cells cultured on the surface of one of the plates (Levesque and Nerem 1985). Shear stress can be calculated based on the flow rate and gap length between the plates. The second one consists of a rotating conical plate creating a homogenous shear stress over a flat plate covered with cultured ('cone-and-plate' apparatus; Schnittler et al. 1993). A shear stress can be calculated based on the plate rotation speed and the length of the gap between the rotating cone and the fixed plate. These two models generate a steady, continuous flow. More recently,

*Corresponding author. Email: fica@kg.ac.rs 
an orbital shear stress model consisting of cell culture wells placed on an orbital shaker rotating at different speeds was used to study the effects of shear stress on endothelial cell morphology, gene expression, and function (Pearce et al. 1996; Kraiss et al. 2000; Yun et al. 2002; Dardik et al. 2005; Potter et al. 2011). Computational models based on computational fluid dynamics were used to describe fluid behavior in rotating cell culture dishes (Berson et al. 2008; Thomas et al. 2011; Salek et al. 2012). These models predicted that orbital shaker generates a flow more closely mimicking the pulsatile flow generated by the beating heart in vivo.

In this paper, we report computational methods for numerical procedures modeling fluid movements, shear stress, and the three-dimensional (3D) free surface of the rotating wave in a orbital shear stress model.

\section{Methods}

\subsection{Computational methods}

\subsubsection{Fluid flow and shear stress}

The simulation is performed using a continuum-based model. The fluid flow is modeled using the finite element method. In order to develop a simple but reliable system to mimic in vitro shear stress, we modeled the conditions of fluid movement in an orbital well shaker system model. Such modeling is relevant to vascular pathologies, as laminar shear stress is atheroprotective for endothelial cells, whereas disturbed or oscillatory shear index (OSI) correlates with the development of atherosclerosis and neointimal hyperplasia. The simulation is performed using the same continuum-based model described earlier. A finite element mesh was generated with 3D brick elements. The total model has about 20,000 3D finite elements. The sensitivity analysis for different mesh density for finite elements was done and the results for shear stress calculation were below $1 \%$ with higher mesh density. Boundary conditions are moving of the bottom orbital surface and rotation of the object around vertical axis. Arbitrary Lagrangian Eulerian (ALE) formulation for mesh moving problem is applied. Free surface mesh algorithm was used.

\subsubsection{Mesh moving algorithm}

In order to make connect blood flow simulation with bioprocess modeling the 3D mesh moving algorithm and ALE formulation for fluid dynamics is applied (Kojic et al. 2008). The governing equations, which include the Navier-Stokes equations of balance of linear momentum and the continuity equation, can be written in the ALE formulation as follows (Filipovic et al. 2006):

$$
\begin{aligned}
\rho\left[v_{i}^{*}+\left(v_{j}-v_{j}^{\mathrm{m}}\right) v_{i, j}\right] & =-p,_{i}+\mu v_{i, j j}+f_{i}^{\mathrm{B}}, \\
v_{i, i} & =0
\end{aligned}
$$

where $v_{i}$ and $v_{i}^{\mathrm{m}}$ are the velocity components of a fluid and of the point on the moving mesh occupied by the fluid particles, respectively; $\rho$ is fluid density, $p$ is fluid pressure, $\mu$ is dynamic viscosity, and $f_{i}^{\mathrm{B}}$ is the body force component which includes gravity force. The symbol ' $*$ ' denotes the mesh-referential time derivative, i.e. the time derivative at a considered point on the mesh,

$$
()^{*}=\left.\frac{\partial()}{\partial t}\right|_{\xi_{i}=\text { const }}
$$

and the symbol ', $i$ ' denotes partial derivative, i.e.

$$
()_{i}=\frac{\partial()}{\partial x_{i}} .
$$

We use $x_{i}$ and $\xi_{i}$ as Cartesian coordinates of a generic particle in space and of the corresponding point on the mesh, respectively. The repeated index means summation, from 1 to 3, i.e. $j=1,2,3$ in Equation (1), and $i=1,2,3$ in Equation (2). In deriving Equation (1), we used the following expression for the material derivative (corresponding to a fixed material point) $\mathrm{D}\left(\rho v_{i}\right) / \mathrm{D} t$ :

$$
\frac{\mathrm{D}\left(\rho v_{i}\right)}{\mathrm{D} t}=\left.\frac{\partial\left(\rho v_{i}\right)}{\partial t}\right|_{\xi}+\left(v_{j}-v_{j}^{\mathrm{m}}\right) \frac{\partial\left(\rho v_{j}\right)}{\partial x_{i}} .
$$

The derivatives on the right-hand side correspond to a generic point on the mesh, with the mesh-referential derivative and the convective term.

Using Equation (5), we obtain from (1) and (2) the system of ordinary differential equations in the form

$$
{ }^{t} \mathbf{M}_{(1)} \mathbf{V}^{*}+{ }^{t} \mathbf{K}_{(1)_{\mathrm{vv}}} \Delta \mathbf{V}+{ }^{t} \mathbf{K}_{\mathrm{vp}} \Delta \mathbf{P}={ }^{t+\Delta t} \mathbf{F}_{(1)}-{ }^{t} \mathbf{F}_{(1)}
$$

and

$$
{ }^{t} \mathbf{M}_{(2)} \mathbf{V}^{*}+{ }^{t} \mathbf{K}_{(2) \mathrm{vv}} \Delta \mathbf{V}={ }^{t+\Delta t} \mathbf{F}_{(2)}-{ }^{t} \mathbf{F}_{(2)},
$$

where matrices $\mathbf{M}_{(1)}$ and $\mathbf{M}_{(2)}$ are mass matrices, $\mathbf{K}_{(1)_{\mathrm{vv}}}$ and $\mathbf{K}_{(2)_{\mathrm{vv}}}$ are viscous, $\mathbf{K}_{\mathrm{vp}}$ is pressure matrix, $\mathbf{F}_{(1)}$ and $\mathbf{F}_{(2)}$ are force matrices with corresponding boundary conditions and body forces. More details of the matrices and vectors are given in (Filipovic et al. 2006).

We modeled free surface tracking where two fluids air and fluid share boundary conditions at the interface.

The methodology of using ALE formulation for free surface problem is well documented in Filipovic et al. (2006) on the benchmark examples such as solitary wave 

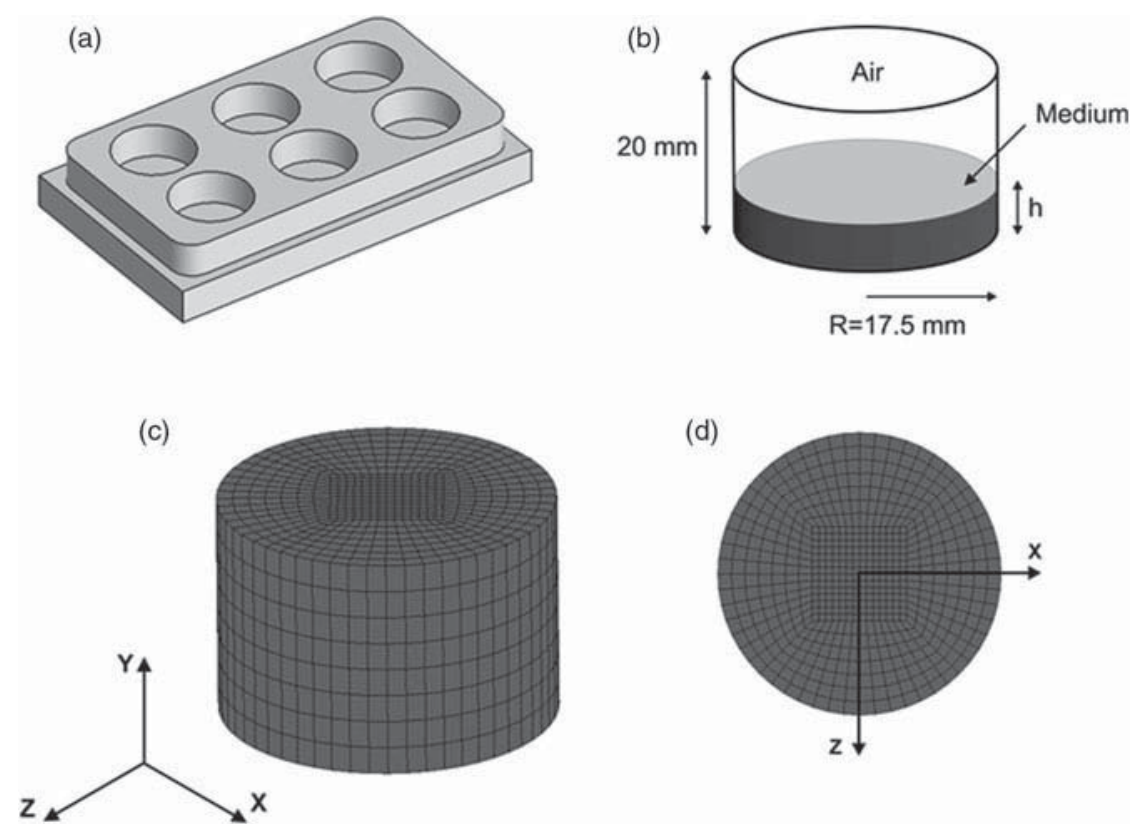

(d)

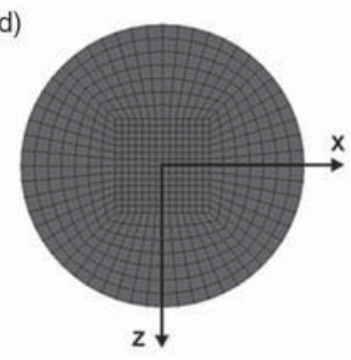

Figure 1. (a) Schematic representation of a six-well culture plate used in the modeling and in the experiments. (b) Schema of a single well geometry. (c) 3D computational mesh for a single well. $X, Y$, and $Z$ denote the stationary reference frame and $x, y$, and $z$ denote the moving reference frame. (d) Cross section in $X Z$ plane.

motion with large displacements of the free boundary and the fluid flow in an infinite contractible and expandable pipe with prescribed large radial wall motion.

\subsubsection{Inclination angle of the surface shear stress}

In addition to the velocity field, the wall shear stress computation is performed. The mean shear stress $\tau_{\text {mean }}$ within a time interval $T$ is calculated as follows (Filipovic et al. 2006, 2011):

$$
\tau_{\text {mean }}=\left|\frac{1}{T} \int_{0}^{T} \mathbf{t}_{\mathrm{s}} \mathrm{d} t\right|,
$$

where $\mathbf{t}_{\mathrm{s}}$ is the surface traction vector and $T$ is the cycle period.
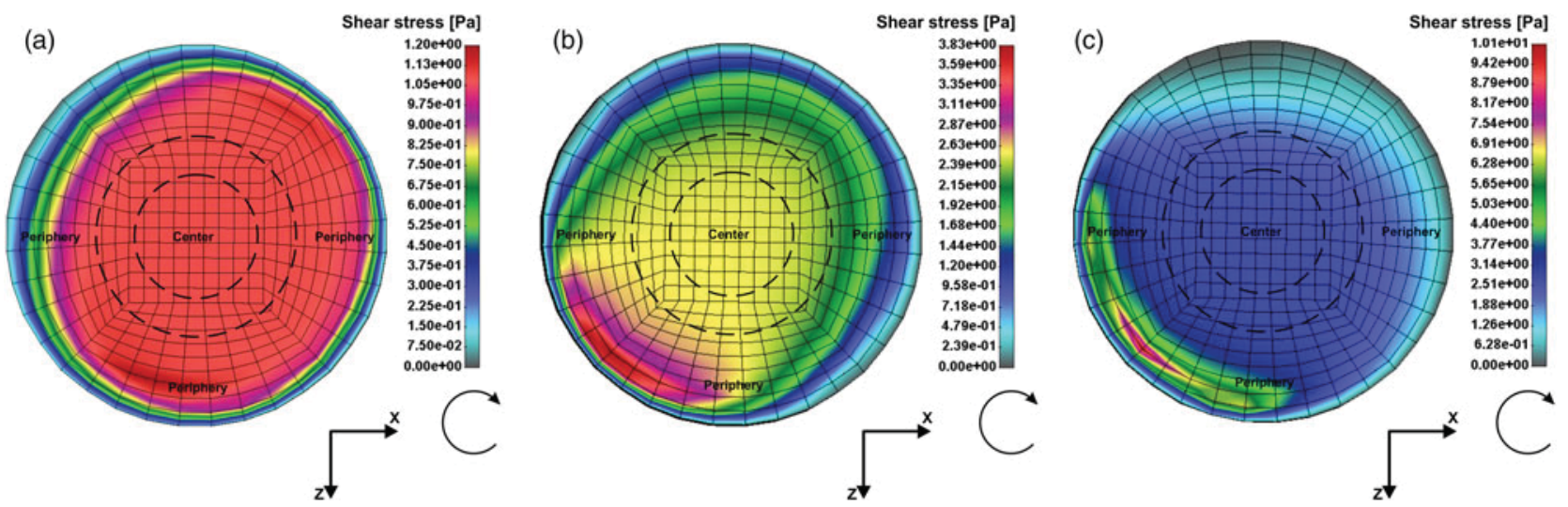

Figure 2. Distribution of shear stress magnitude on the bottom face of a single well at two different rotation speeds. (a) 100, (b) 150 , and (c) $200 \mathrm{rpm}$. Filling volume of the well is $2 \mathrm{ml}(h=2.1 \mathrm{~mm})$. The magnitude of the shear stress is color-coded (blue, low; green-yellow, intermediate; pink-red, high). Values are given in Pa. The views of the distributions are from the top. 
Another scalar quantity is a time-averaged magnitude of the surface traction vector, calculated as

$$
\tau_{\text {mag }}=\frac{1}{T} \int_{0}^{T}\left|\mathbf{t}_{\mathrm{s}}\right| \mathrm{d} t,
$$

Also, a very important scalar in the quantification of unsteady blood flow is the OSI defined as (Ku et al. 1985)

$$
\mathrm{OSI}=\frac{1}{2}\left(1-\frac{\tau_{\mathrm{mean}}}{\tau_{\mathrm{mag}}}\right) .
$$

The inclination angle of the surface shear stress is calculated as angle between main axes of the surface traction vector.

\subsubsection{Drag force}

Drag force was calculated from direct integration of the pressure and wall shear stress distributions on the surfaces of the aortic wall:

$$
\sum F=\int p \mathrm{~d} A+\int \tau \mathrm{d} A+F_{\mathrm{g}}
$$

where the integrals are surface finite elements along surface of bottom surface of well and $F_{\mathrm{g}}$ is gravity force.

\subsubsection{Orbital shaker model}

The schema of culture plate for six identical wells is shown in Figure 1(a). Each well is $20 \mathrm{~mm}$ deep and has a radius of $R=17.5 \mathrm{~mm}$ (Figure 2(b)). The wells are mounted on an orbital shaker and undergo a two-dimensional linear translation in the horizontal plane (i.e., $X Z$ plane). Each well is filled with $2 \mathrm{ml}$ of liquid medium. The Reynolds number remains in the laminar regime. The orbital radius is $38 \mathrm{~mm}$ and orbital speeds are 100, 150, and $200 \mathrm{rpm}$. Computational mesh for a single well as well as the stationary and moving reference frame is presented in Figure 1(c),(d). Magnitude of the shear stress in given in Pa.

\subsection{Experimental Procedure}

\subsubsection{Endothelial cell culture}

Human umbilical vein endothelial cells (HUVECs) were prepared and cultured as described (Bezzi et al. 2003) and were used between passages 3 and 5. HUVECs were cultured in M199 medium (Lonza, Basel, Switzerland) supplemented with $10 \%$ heat-inactivated fetal calf serum (Chemie Brunschwig AG, Basel, Switzerland), $12 \mu \mathrm{g} / \mathrm{ml}$ of bovine brain extract (Lonza), $10 \mathrm{ng} / \mathrm{ml}$ human recombinant epidermal growth factor (Genzyme, Baar, Switzerland), $25 \mathrm{U} / \mathrm{ml}$ heparin, $1 \mu \mathrm{g} / \mathrm{ml}$ hydrocortisone (Sigma-Aldrich Chemie $\mathrm{GmbH}$, Buchs, Switzerland), $2 \mathrm{mM}$ L-glutamine,
$100 \mu \mathrm{g} / \mathrm{ml}$ streptomycin, and $100 \mathrm{U} / \mathrm{ml}$ penicillin (Life Technologies, Basel, Switzerland). According to the experimental needs, culture dishes for HUVECs were pre-coated either with $0.5 \%$ gelatin or $3 \mu \mathrm{g} / \mathrm{ml}$ fibronection or $1 \mu \mathrm{g} / \mathrm{ml}$ collagen for $3-4 \mathrm{~h}$ at $37^{\circ} \mathrm{C}$. HUVECs were passaged every 3 days at a 1:3 ratio. For detachment, 0.05\% trypsinethylenediaminetetraacetic acid (EDTA) was used. Cells were cultured at $37^{\circ} \mathrm{C}$ and $5 \% \mathrm{CO}_{2}$.

\subsubsection{Shear stress studies}

A volume of $5 \times 10^{5}$ HUVECs were seeded to confluence in six-well cell culture dishes (TPP Techno Plastic, Trasadingen, Switzerland) in $2 \mathrm{ml}$ medium. After $8 \mathrm{~h}$, the cell culture plate was fixed on a horizontal shaker system (Vibromix 314 EVT, Tehtnica, Železniki, Slovenia) with an orbit of $38 \mathrm{~mm}$ at $150 \mathrm{rpm}$ to generate orbital shear stress. After $24 \mathrm{~h}$ of orbital shaking, plates were collected for further analysis (imaging and Western blotting) and compared with cultures maintained under static conditions or to cultures exposed to a corresponding laminar flow in a cone-and-plate apparatus (Buschmann et al. 2005).

\subsubsection{Preparation of cell lysates}

HUVEC cultures were washed in ice-cold phosphatebuffered saline (PBS). In the orbital shaking model, the cells at the center of the well were removed with cell scraper and washed away with PBS, leaving the periphery of the cultures intact. Lysis buffer was added to the peripheral cells in the well or to the cells previously collected from the center of the well. In the static cell, cultures and cone-andplates system all cells were collected. Cells were lysed in $0.75 \mathrm{ml}$ of lysis buffer A $(20 \mathrm{mM}$ Tris $-\mathrm{HCl}, \mathrm{pH} 7.6$, $150 \mathrm{mM} \mathrm{NaCl}, 1 \mathrm{mM}$ EDTA, 1 mM EGTA, $2.5 \mathrm{mM}$ sodium pyrophosphate, $1 \mathrm{mM} \beta$-glycerophosphate, $1 \mathrm{mM}$ sodium vanadate, and 1\% Triton X-100 (Sigma Chemie, Buochs, Switzerland). Cell lysates were clarified by spinning at $13,000 \mathrm{~g}$ for $15 \mathrm{~min}$ at $4^{\circ} \mathrm{C}$. Protein content of each sample was measured by using a Bio-Rad detergent-compatible assay (Bio-Rad, Richmond, CA, USA).

\subsubsection{Western blot analysis}

Cell lysates $(15 \mu \mathrm{g}$ of protein per well) was heated for 4 min at $93^{\circ} \mathrm{C}$ and resolved by $9 \%$ sodium dodecyl sulfate polyacrylamide gel electrophoresis. After electrophoresis, separated proteins were transferred to Immobilon-P transfer membrane (Millipore, Zug, Switzerland) in $1 \times$ transfer buffer (25 mM Tris, $192 \mathrm{mM}$ glycine, 10-20\% methanol) for $70 \mathrm{~min}$ at $100 \mathrm{~V}$. After transfer, membranes were blocked by $5 \%$ bovine serum albumin- Tris-buffered saline Tween $(10 \mathrm{mM}$ Tris, $150 \mathrm{mM} \mathrm{NaCl}, 0.1 \%$ Tween20, $\mathrm{pH} 7.4$ ) at room temperature for $1 \mathrm{~h}$. Membranes were 

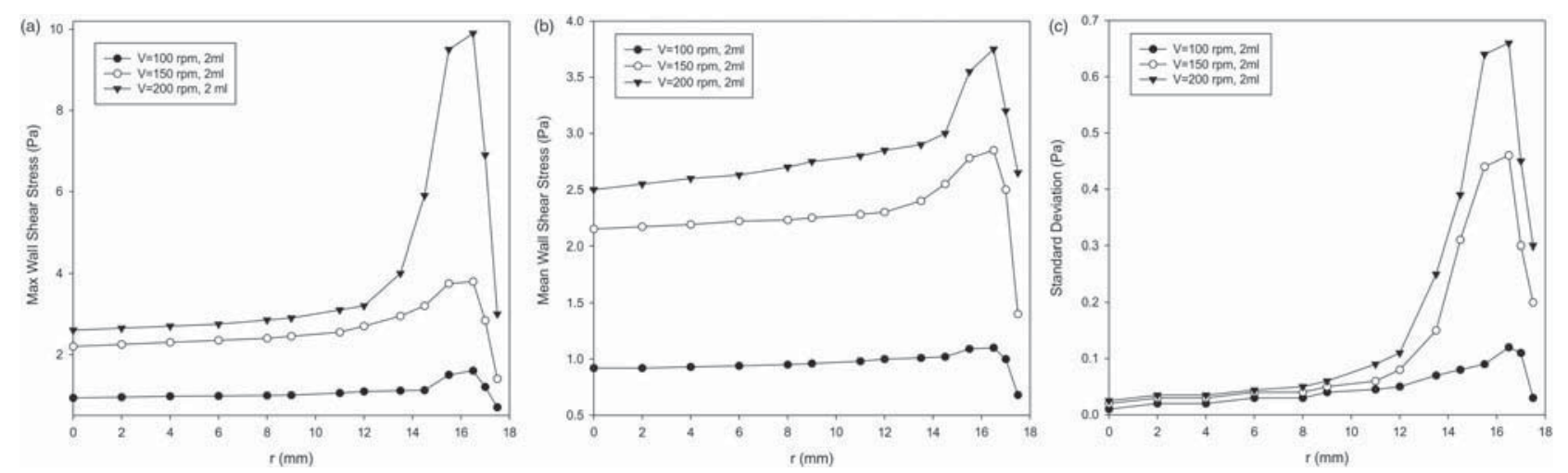

Figure 3. Summary of the radial distribution of the wall shear stress magnitude for rotation speeds of 100, 150, and 200 rpm, as a function of the radial location from the center of the well (in $\mathrm{mm}$ ): (a) maximum wall shear stress intensity; (b) mean wall shear magnitude; and (c) standard deviation of the shear stress fluctuations. Values are given in Pa.

incubated overnight with primary antibodies. Next day, membranes were washed three to four times with TBS-T, and then incubated with peroxidase-conjugated secondary antibodies for $1 \mathrm{~h}$. Blots were incubated with Luminata Western HRP Substrate for 1 min, excess substrate wiped with tissue paper and the chemiluminescence signal was detected using X-ray films or using FluorChem E imager (Bio-Techne, MN, USA). The antibodies used were as follows: anti-Kruppel-like Factor 2 (KLF-2) and antieNOS from Cell Signaling Technology (Beverly, MA, USA); anti-COX-2 from Cayman Chemical Company (Ann Arbor, MI, USA); anti-actin from Sigma Chemie (Buochs, Switzerland).

\section{Results}

\subsection{Numerical results}

First, we modeled shear stress distribution for the bottom surface of a single well $(R=17.5 \mathrm{~mm})$ of a six-well cell culture plate filled with $2 \mathrm{ml}$ medium $(h=2.1 \mathrm{~mm})$ for different orbital velocities, i.e., 100, 150, and $200 \mathrm{rpm}$ (Figure 1(a),(b)). The stationary reference frame computational mesh for a single well is shown in Figure 1(c),(d) with $X, Y$, and $Z$ denoting the stationary reference frame and $x, y$, and $z$ denoting the moving frame. Finite element mesh with cubic eight-node element is used. The cross section in $X Z$ plane for bottom surface with finite element

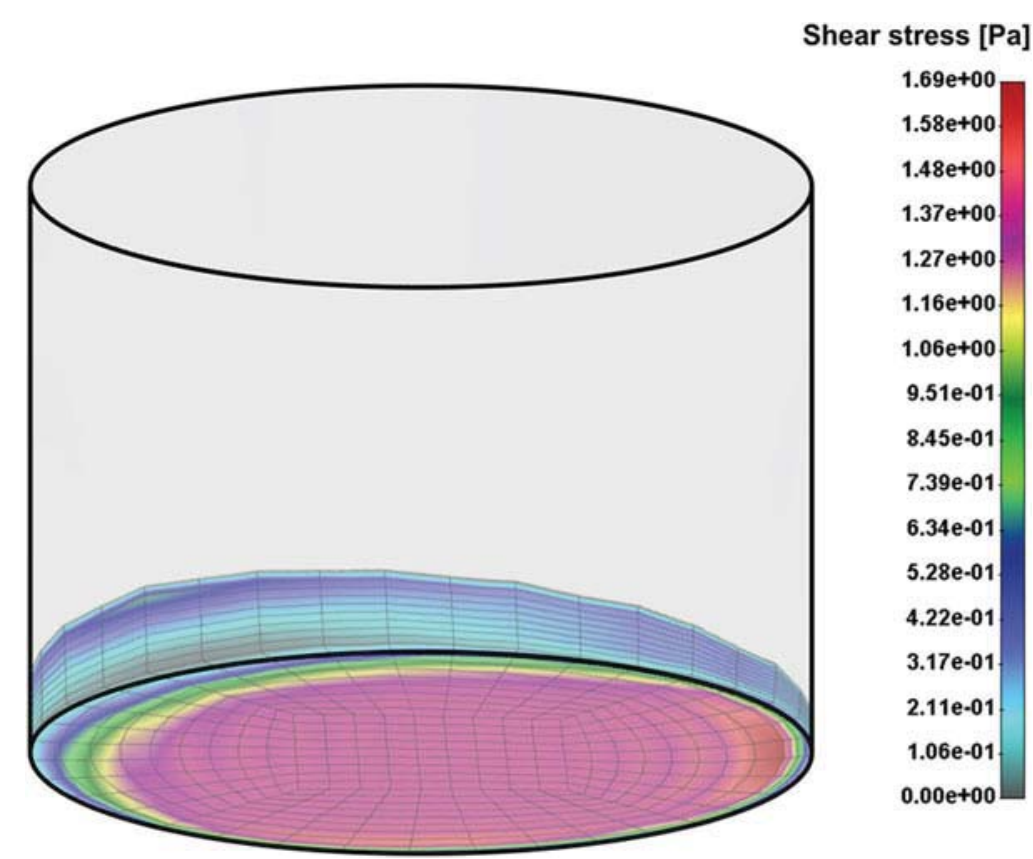

Figure 4. 3D view of free surfaces for 150-rpm orbital speed. The well is filled with $2 \mathrm{ml}$ volume. At high angular velocity the liquid medium generates a wave on the free surface. High shear stress is located underneath the leading edge of the wave, while lower shear stress is located following the peak of the wave. Values are given in $\mathrm{Pa}$. 


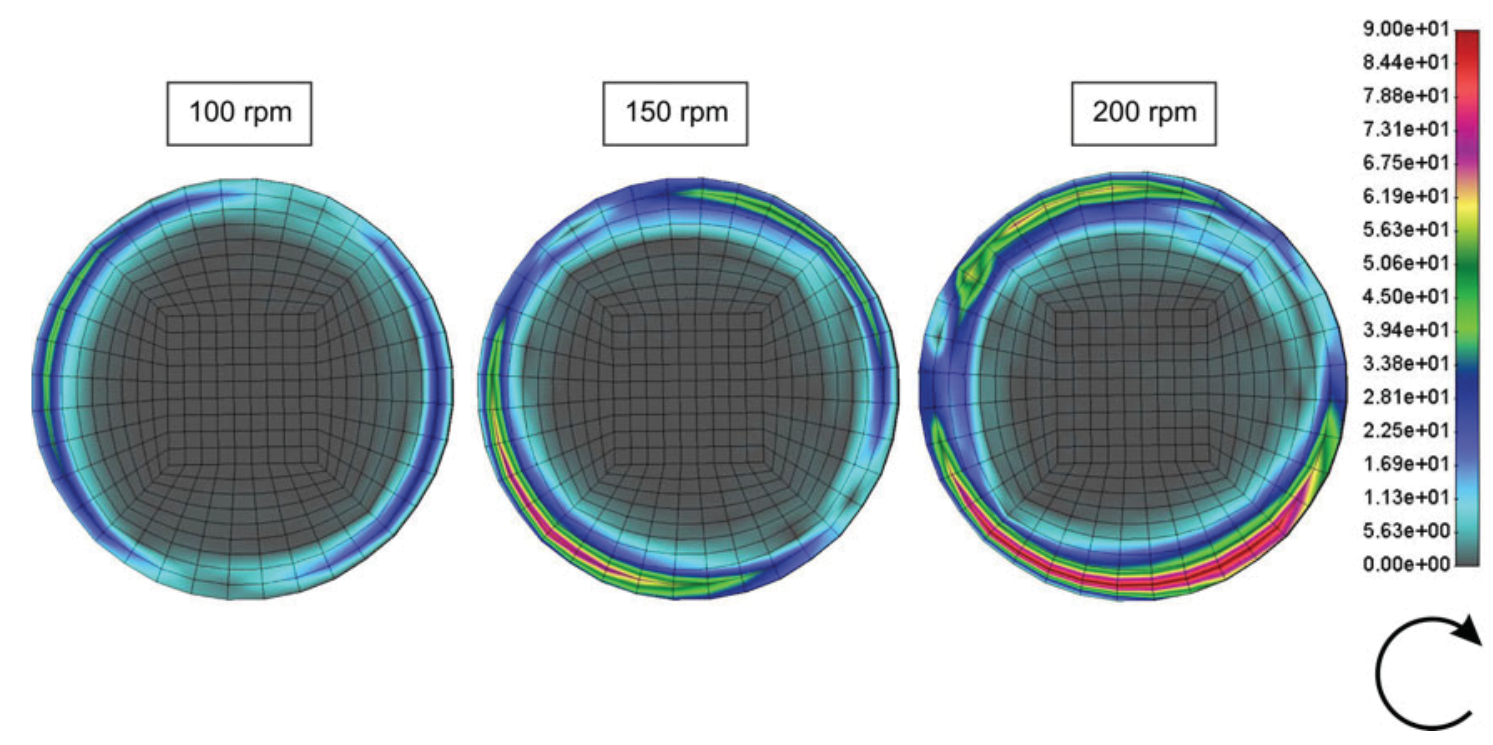

Figure 5. Distribution of the inclination angle of the surface shear stress vectors at the indicated orbital speeds measured relative to the bottom surface. The inclination angle is color-coded (blue, low; green-yellow, intermediate; pink-red, high). Values are given in degrees (0-90).

mesh is presented in Figure 1(d). Boundary conditions for rotation are imposed for the whole well where free surface calculation is used for each time step. Rotation cycle is divided into 500 time steps. Similar results where obtained when using different numbers of time steps (data not shown). For $100 \mathrm{rpm}$ the wall shear stress is nearly constant over the entire well, with only a small oscillatory increase toward the periphery and lowest values along the side wall. The highest calculated value is $<2 \mathrm{~Pa}$ (Figure 2 (a)). For higher rotation rates (150 and $200 \mathrm{rpm})$, the

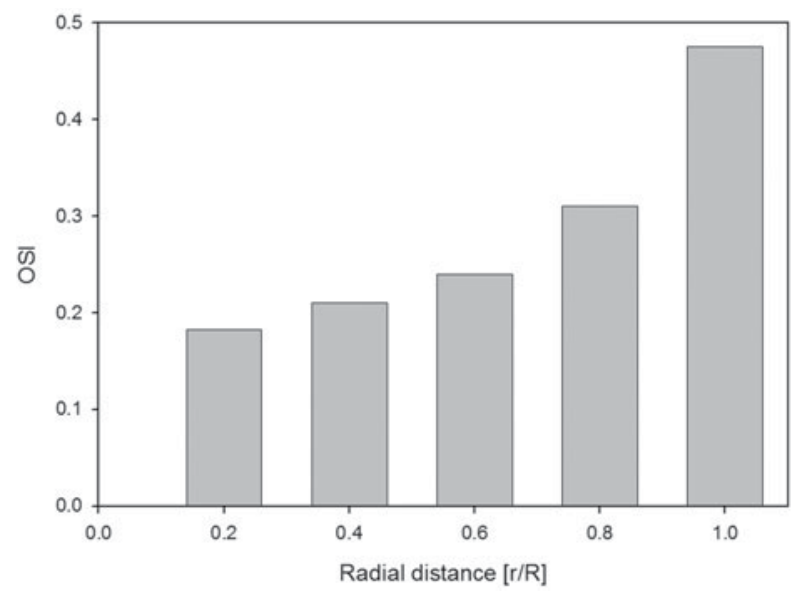

Figure 6. Distribution of oscillatory shear index for 200-rpm orbital speed (OSI, oscillatory shear index). The wall shear stress is nonuniform and with higher circular component in the periphery of the well, while it is more uniform and with smaller circular component in the center. Scale gives values for OSI: 0.5 , maximal oscillatory flow; 0 , no oscillatory flow. distribution of the shear stress at the bottom of the well is more complex and nonuniform, with the highest calculated value toward the periphery of the well (around $4 \mathrm{~Pa}$ at $150 \mathrm{rpm}$ and around $10 \mathrm{~Pa}$ at $200 \mathrm{rpm}$ ) and lowest values against the side wall. These values are in the range of those occurring in vessels in the venous and arterial circulations. At 150 and $200 \mathrm{rpm}$ the fluid behaves in an wave-like manner, with a wave of high shear stress followed by an opposed wave of low shear stress rotating at an angular velocity equal to the orbital velocity of the well (Figure 2 (b),(c)). The radial distribution of the maximal and mean values of the wall shear stress as a function of the radial location for different orbital velocity (100, 150, and $200 \mathrm{rpm}$ ) is presented in Figure 3(a),(b). This confirms that the maximal values are predicted at the periphery. The shear stress, however, drops when approaching the sidewall because of zero velocity at the sidewall. The standard deviations of the shear stress fluctuations for the different rotation velocities are presented in Figure 3(c). The high shear stress fluctuations at 150 and $200 \mathrm{rpm}$ are due to the wave-like oscillations of the shear stress at the periphery of the well at these rotation speeds. These oscillatory fluctuations of the shear stress are due to the fact that at high angular velocities the liquid medium generates a wave on the free surface, as represented by a $3 \mathrm{D}$ view of free surfaces modeled at a rotation speed of $150 \mathrm{rpm}$ and a filling volume of $2 \mathrm{ml}$ (Figure 4). The region of high shear stress formed underneath the leading edge of the moving wave is due to the reduce liquid height by constant velocity and results in a high velocity/distance ratio. The region of lower stress is located following the peak of the wave height and is due to the low velocity/ 


\section{Drag force [N]}

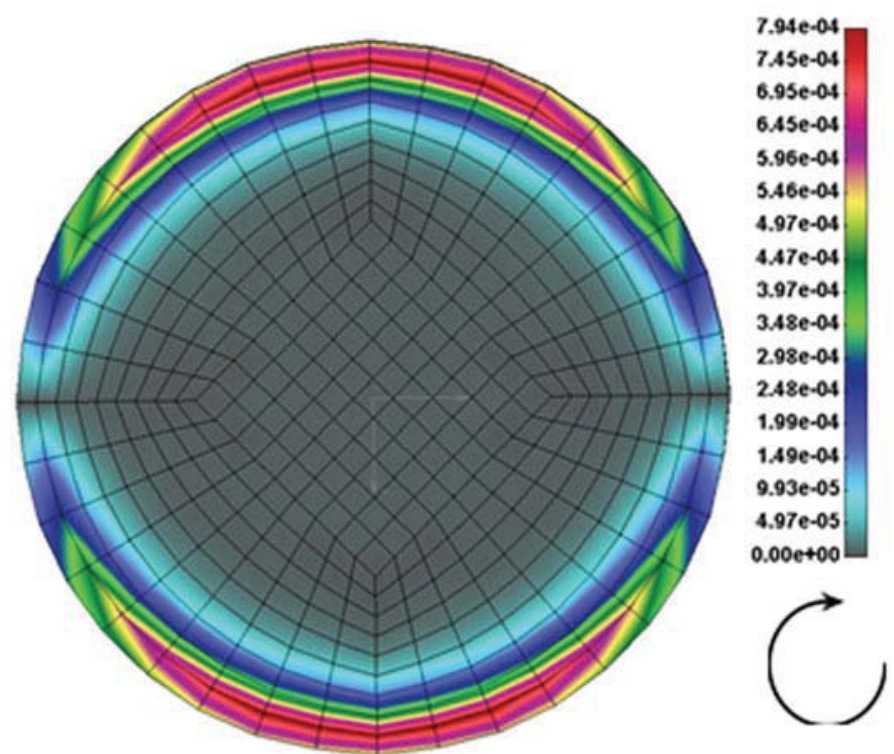

Figure 7. Distribution of drag forces. High drag force values are calculated for the well periphery while lower values are calculated for the central par of the bottom surface of the well. Scale gives the intensity of the force in N. Rotation is indicated by the arrow.

(a)

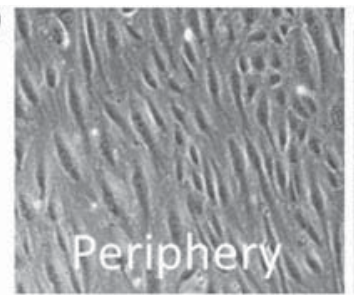

(b)

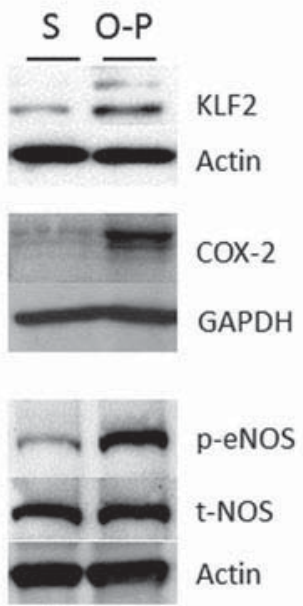

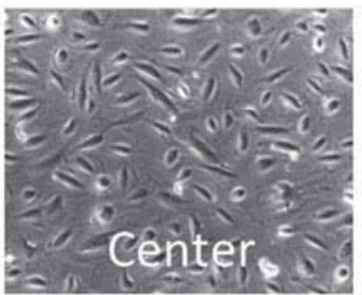

(c)
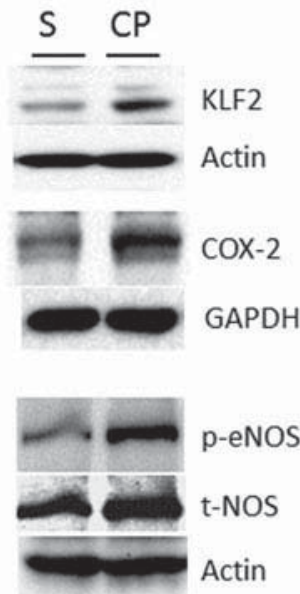

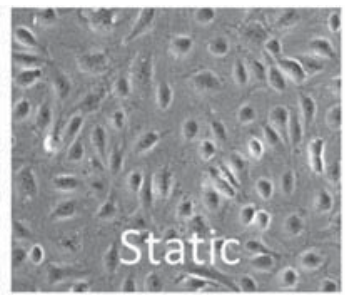

(d)
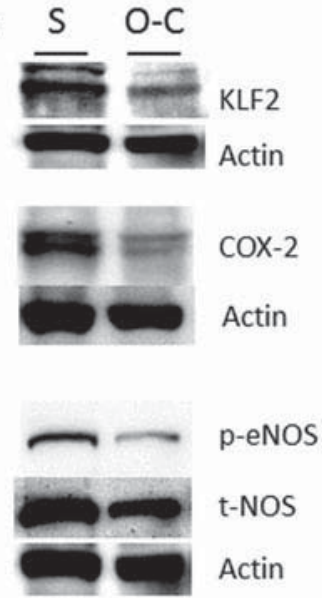

Figure 8. Morphological and biochemical responses of HUVECs to shear stress. (a) Morphology of HUVECs after $24 \mathrm{~h}$ of orbital shear stress in the periphery of the well (left panel) or in the center of the well (mid panel). HUVECs under static conditions are shown for comparison in the right panel. Orbital shear stress in the periphery of the well causes HUVECs to align in the direction of flow. (b-d) Western blot analysis of the effect of shear stress on KLF-2 and COX-2 expression and eNOS phosphorylation. Confluent monolayers of HUVECs were exposed for $24 \mathrm{~h}$ to orbital shaking in the periphery of the well (b, O-P), to homogenous laminar shear stress in the coneand-plate apparatus (c, CP) or to orbital shaking in the center of the well (c, O-C). Experimental conditions are compared with HUVECs cultured under static conditions $(n=3)$. 
distance ratio. Distribution of the inclination angle of the surface shear stress vectors measured relative to the bottom well surface is presented in Figure 5. It can be observed that inclination angle values are higher in the periphery of the well (approaching $90^{\circ}$ at 150 and $200 \mathrm{rpm}$ ), while they are close to zero in the central portion of the well.

The distribution of oscillatory shear index is presented in Figure 6. The wall shear stress is high and more nonuniform in the periphery of the well, with a high circular component, resulting in a high oscillatory shear index. In contrast, uniform and smaller values of wall shear stress are calculated for the center of the well with smaller circular components of wall shear stress and smaller oscillatory shear indexes. Similar results for directional oscillatory shear index were obtained by Chakraborty et al. (2012). Distribution of drag force taking into account viscous force and the force from fluid pressure is presented in Figure 7. High and fluctuating drag force values are predicted for the periphery of the bottom surface, while uniform and low drag forces, approaching zero value, are predicted for the central part of the bottom surface.

\subsection{Experimental results}

The above-mentioned mathematical modeling predicts a high wave-like shear stress at the periphery of the well, and a more constant shear stress at the center of the well. To experimentally validate these predictions, we seeded HUVECs in $35 \mathrm{~mm}$ diameter wells of a six-well cell culture dishes in $2 \mathrm{ml}$ medium. Cells were allowed to attach for $8 \mathrm{~h}$ and then exposed to orbital shaking at $150 \mathrm{rpm}$. Control cells were maintained under static conditions or exposed to a homogenous laminar shear stress using a cone-and-plate apparatus (Buschmann et al. 2005).

After $24 \mathrm{~h}$ of orbital shaking, micrographs of cells were taken at the center and at the periphery of the well. HUVECs at the periphery showed an elongated morphology aligned with the flow (Figure 8(a), left panel), while HUVECs in the center of the well failed to align, showed an irregular morphology with signs of rounding, retraction and, in some cases, detachment (Figure 8(a), mid panel). HUVECs cultured under static conditions with the typical cobblestone morphology are shown for comparison (Figure 8(a), right panel). Thereafter, cells at the periphery of the well (outer $7.5 \mathrm{~mm}$ radius) or at the center (inner $5 \mathrm{~mm}$ radius) were collected for Western blotting analysis. Compared with HUVECs cultured under static conditions, cells from the periphery of the well expressed increased levels of Kruppel-like Factor 4 (KLF4), COX-2, and phospho-eNOS (Figure 8(b)): three effects known to be induced by laminar shear stress (Topper et al. 1996; Hamik et al. 2007). Similar effects were observed in
HUVECs exposed to homogenous shear stress using the cone-and-plate apparatus (Figure 8(c)). In contrast, Western blotting analysis showed that cells collected from the center of the well had lower expression levels of KLF-4, COX-2, and phospho-eNOS (Figure 8(d)).

\section{Discussion}

Shear stress is critical to modulate vascular endothelial cell functions and fate. For in vitro experimental studies, it is therefore important to model different conditions of shear stress, intensity, and type. The orbital shaker model has been used in the past and is being considered as a possible alternative to classical systems, in particular the parallel plates and cone-and-plate systems. Exact modeling of the hemodynamic conditions in the orbital shaker system is essential in order to understand its characteristics and possible limitations. Based on the numerical modeling reported here and on previous studies it emerges that orbital shaking generates different types of shear stress in different regions of the well leading to dramatically different biological responses. At the periphery of the well, shear stress is oscillatory with wave-like fluctuations, higher drag forces and a higher inclination angle. At the center of the well, shear stress is of lower intensity and with a lower inclination angle, without oscillations and almost zero drag force. Thus, in the periphery, the hydrodynamic conditions reflect the pulsatile character of the blood flow in vivo with an important drag force. The effect on KLF-4 and COX-2 expression and eNOS phosphorylation is similar to that observed for the parallel plate and the cone-and-plate models. In contrast, endothelial cell close to the center of the well responded differently and showed evidence of cell suffering.

The methods for numerical modeling used here describe fluid equations and a mesh-moving algorithm at different orbital speeds (100, 150, and $200 \mathrm{rpm})$ and 3D free surface constructions of the rotating wave including shear stress inclination angle, OSI, and drag forces, which have not been previously reported in other models. We show that the fluid motion and the wall shear stress distribution within a cylindrical well undergoing orbital translation in a horizontal plane are controlled by the orbital radius of gyration and by the angular speed. For 100-rpm angular speed, the wall shear stress is nearly constant over the entire well with highest values below $2 \mathrm{~Pa}$. For higher rotation rates, distribution of the wall shear stress is much more complex and nonuniform at the bottom surface. The maximum predicted shear stress for 150- and 200-rpm angular speed approaches 4 and $10 \mathrm{~Pa}$ at the periphery of the well, respectively. This computational fluid dynamic analysis predicts that shear stress distribution for orbital shaker is not constant when moving from the center to the periphery of the well. 
Through experimental validation, we verified that the orientation of endothelial cells and the modulation of gene expression and signaling events are indeed related to the flow topology, type, and intensity of the wall shear stress. Endothelial cells at the periphery of the well reacted with alignment in the direction of fluid flow and with increased expression of COX-2 and KLF-4 and eNOS phosphorylation, as expected for cells exposed to physiological laminar shear stress. In contrast, endothelial cells in the center of the well failed to align, downregulated COX-2 and KLF-4 expression and eNOS phosphorylation as expected for cells exposed to non-physiological shear stress. In addition, they showed signs of suffering such as retraction, rounding up, and detachment. Definition and calculation of the inclination angle, OSI, and drag force provide more insight to the understanding of the endothelial cell behavior under different flow conditions. The predicted wave-like, pulsatile character of flow with higher drag forces and inclination angle at the periphery are likely to mimic more closely physiological conditions for endothelial cells as observed in vessels. Thus, this numerical modeling predicts complex and heterogeneous shear stress conditions in an orbital shaker model with higher shear stress with wave-like, pulsatile character, higher drag force values, higher circular component, and larger inclination angle at periphery and more uniform, lower shear stress with smaller drag force, circular component, and inclination angle at the center of the well. The inclination angle and drag force as well as the oscillatory index calculated in our work are new quantitative values, which gives better understanding of the hydrodynamic conditions in this system.

This model mimics two important clinically relevant features. First, it generates wave-like, pulsatile flow similar to the pulsatile character of the blood flow in large and medium size elastic arteries in vivo. The cone-andplate and the parallel plates models generate an ideal, continuous laminar flow, which does not exists in large and medium size arteries in vivo. It is important to further characterize this model by comparing the cellular and molecular responses of endothelial cells to continuous, versus wave-like, pulsatile flows. Second, this model allows exposing a homogenous population of endothelial cells to two different shear stresses under the same experimental setting. This mimics the conditions of physiological versus nonphysiological shear stresses observed in relative close proximity within the same vessel in vivo, e.g., at sites of bifurcations, stenoses, dilatations, or atherosclerotic plaques. Being able to reproduce such different conditions in the same culture allows investigating responses to different shear stresses under identical biological and biochemical conditions.

In conclusion, we have reported the modeling of the shear stress distribution in an orbital shaker model using computational fluid dynamics with finite elements method.
These modeling and the performed experimental validation indicate that the orbital shaker model is an important complement to the classical parallel plates and cone-and-plate models. Additional characterization of the endothelial cell responses in different regions of the well is necessary in order to better describe the model. A systematic comparison with the parallel plates and cone-and-plate models might reveal important differences in the way endothelial cells respond to wave-like, pulsatile flow mimicking the pulsatile character of the blood flow in vivo compared to continuous flow.

\section{Acknowledgements}

The authors thank Dr. Jochen Seebach and Prof. Hans-Joachim Schnittler, University of Münster, Germany, for providing samples of cone-and-plate experiments.

\section{Disclosure statement}

No potential conflict of interest was reported by the author(s).

\section{Funding}

This study was funded by a SCOPES grant from the Swiss National Science Foundation [grant number IZ74Z0_137357], the Medic foundation, the SmallArtey (SMART) Marie Curie Initial Training Network of the European Commission, and grants from Serbian Ministry of Science [grant number III41007], [grant number ON174028].

\section{References}

Ballermann BJ, Dardik A, Eng E, Liu A. 1998. Shear stress and the endothelium. Kidney Int Suppl. 54(s67):100-108. doi:10.1046/j.1523-1755.1998.06720.x.

Berson RE, Purcell MR, Sharp MK. 2008. Computationally determined shear on cells grown in orbiting culture dishes. Adv Exp Med Biol. 614:189-198. doi:10.1007/978-0-38774911-2_22.

Bezzi M, Hasmim M, Bieler G, Dormond O, Ruegg C. 2003. Zoledronate sensitizes endothelial cells to tumor necrosis factor-induced programmed cell death: evidence for the suppression of sustained activation of focal adhesion kinase and protein kinase B/Akt. J Biol Chem. 278(44):43603-43614. doi:10.1074/jbc.M308114200.

Buschmann MH, Dieterich P, Adams NA, Schnittler HJ. 2005. Analysis of flow in a cone-and-plate apparatus with respect to spatial and temporal effects on endothelial cells. Biotechnol Bioeng. 89(5):493-502. doi:10.1002/bit.20165.

Cai W, Schaper W. 2008. Mechanisms of arteriogenesis. Acta Biochim Biophys Sin (Shanghai). 40(8):681-692. doi:10.1111/j.1745-7270.2008.00436.x.

Chakraborty A, Chakraborty S, Jala V, Haribabu B, Sharp MK, Berson RE. 2012. Effects of biaxial oscillatory shear stress on endothelial cell proliferation and morphology. Biotechnol Bioeng. 109(3):695-707. doi:10.1002/bit.24352.

Chiu JJ, Chien S. 2011. Effects of disturbed flow on vascular endothelium: pathophysiological basis and clinical perspec- 
tives. Physiol Rev. 91(1):327-387. doi:10.1152/physrev. 00047.2009.

Dardik A, Chen L, Frattini J, Asada H, Aziz F, Kudo FA, Sumpio BE. 2005. Differential effects of orbital and laminar shear stress on endothelial cells. J Vasc Surg. 41(5):869-880. doi:10.1016/j.jvs.2005.01.020.

Filipovic N, Mijailovic S, Tsuda A, Kojic M. 2006. An implicit algorithm within the Arbitrary Lagrangian-Eulerian formulation for solving incompressible fluid flow with large boundary motions. Comp Methods Appl Mech Eng. 195(44-47):6347-6361. doi:10.1016/j.cma.2005.12.009.

Filipovic N, Milasinovic D, Zdravkovic N, Böckler D, von Tengg-Kobligk H. 2011. Impact of aortic repair based on flow field computer simulation within the thoracic aorta. Comput Methods Programs Biomed. 101(3):243-252. doi:10.1016/j.cmpb.2011.01.005.

Folkman J, Haudenschild CC, Zetter BR. 1979. Long-term culture of capillary endothelial cells. Proc Natl Acad Sci USA. 76(10):5217-5221. doi:10.1073/pnas.76.10.5217.

Hamik A, Lin Z, Kumar A, Balcells M, Sinha S, Katz J, Feinberg MW, Gerszten RE, Edelman ER, Jain MK. 2007. Kruppellike factor 4 regulates endothelial inflammation. J Biol Chem. 282(18):13769-13779. doi:10.1074/jbc.M700078200.

Kojic M, Filipovic N, Stojanovic B, Kojic N. 2008. Computer modeling in bioengineering: theoretical background, examples and software. Chichester: Wiley.

Kraiss LW, Weyrich AS, Alto NM, Dixon DA, Ennis TM, Modur V, McIntyre TM, Prescott SM, Zimmerman GA. 2000. Fluid flow activates a regulator of translation, p70/p85 S6 kinase, in human endothelial cells. Am J Physiol Heart Circ Physiol. 278(5):H1537-H1544.

Ku DN, Giddens DP, Zarins CK, Glasov S. 1985. Pulsatile flow and atherosclerosis in the human carotid bifurcation. Atherosclerosis. 5:293-302.

Levesque MJ, Nerem RM. 1985. The elongation and orientation of cultured endothelial cells in response to shear stress. J Biomech Eng. 107(4):341-347. doi:10.1115/1.3138567.

Nerem RM, Alexander RW, Chappell DC, Medford RM, Varner SE, Taylor WR. 1998. The study of the influence of flow on vascular endothelial biology. Am J Med Sci. 316(3):169-175. doi:10.1097/00000441-199809000-00004.

Paszkowiak JJ, Dardik A. 2003. Arterial wall shear stress: observations from the bench to the bedside. Vasc Endovascular Surg. 37(1):47-57. doi:10.1177/153857440303700107.

Pearce MJ, McIntyre TM, Prescott SM, Zimmerman GA, Whatley RE. 1996. Shear stress activates cytosolic phospholipase A2(cPLA2) and MAP kinase in human endothelial cells. Biochem Biophys Res Commun. 218(2):500-504. doi:10.1006/bbrc.1996.0089.

Potter CM, Lundberg MH, Harrington LS, Warboys CM, Warner TD, Berson RE, Moshkov AV, Gorelik J, Weinberg PD, Mitchell JA. 2011. Role of shear stress in endothelial cell morphology and expression of cyclooxygenase isoforms. Arterioscler Thromb Vasc Biol. 31(2):384-391. doi:10. 1161/ATVBAHA.110.214031.

Salek MM, Sattari P, Martinuzzi RJ. 2012. Analysis of fluid flow and wall shear stress patterns inside partially filled agitated culture well plates. Ann Biomed Eng. 40(3):707-728. doi:10.1007/s10439-011-0444-9.

Schnittler HJ, Franke RP, Akbay U, Mrowietz C, Drenckhahn D. 1993. Improved in vitro rheological system for studying the effect of fluid shear stress on cultured cells. Am J Physiol. 265(1 Pt 1):C289-C298.

Schor AM, Schor SL, Allen TD. 1983. Effects of culture conditions on the proliferation, morphology and migration of bovine aortic endothelial cells. J Cell Sci. 62:267-285

Sumpio BE, Timothy Riley J, Dardik A. 2002. Cells in focus: endothelial cell. Int J Biochem Cell Biol. 34(12):1508-1512. doi:10.1016/S1357-2725(02)00075-4.

Thomas JM, Chakraborty A, Sharp MK, Berson RE. 2011. Spatial and temporal resolution of shear in an orbiting petri dish. Biotechnol Prog. 27(2):460-465. doi:10.1002/btpr. 507.

Topper JN, Cai J, Falb D, Gimbrone, Jr., MA. 1996. Identification of vascular endothelial genes differentially responsive to fluid mechanical stimuli: cyclooxygenase-2, manganese superoxide dismutase, and endothelial cell nitric oxide synthase are selectively up-regulated by steady laminar shear stress. Proc Natl Acad Sci USA. 93(19):10417-10422. doi:10.1073/pnas.93.19.10417.

Warboys CM, Eric Berson R, Mann GE, Pearson JD, Weinberg PD. 2010. Acute and chronic exposure to shear stress have opposite effects on endothelial permeability to macromolecules. Am J Physiol Heart Circ Physiol. 298(6): H1850-H1856. doi:10.1152/ajpheart.00114.2010.

Yun S, Dardik A, Haga M, Yamashita A, Yamaguchi S, Koh Y, Madri JA, Sumpio BE. 2002. Transcription factor Sp1 phosphorylation induced by shear stress inhibits membrane type 1-matrix metalloproteinase expression in endothelium. J Biol Chem. 277(38):34808-34814. doi:10.1074/jbc. M205417200. 